\title{
Interdisciplinaridade e integração pesquisa, ensino e extensão na promoção da saúde: a comunicação social na saúde da comunidade e educação básica
}

\author{
Isciplinarity and integration of teaching, research and extension \\ n health promotion: social communication in community \\ health and basic education
}

Miria Suzana Burgos', Gabriela Maria Etges', Priscila Tatiana da Silva', Daniel Rocha', Samuel Aguiar ${ }^{1}$ 'Universidade de Santa Cruz do Sul (UNISC), Santa Cruz do Sul, RS, Brasil.

Recebido em: Dezembro 2015 / Aceito em: Dezembro 2015 mburgos@unisc.br

\section{RESUMO}

Objetivo: descrever o uso dos recursos de Comunicação Social pelos docentes, pesquisadores, mestrandos e mestres do programa de Pós-Graduação em Promoção da Saúde (PPGPS), da Universidade de Santa Cruz do Sul (UNISC), de parte das atividades de pesquisa, ensino e extensão, voltadas à saúde na comunidade e educação básica. Método: para a coleta de dados, foram revisados onze informativos e uma parcela dos projetos (10), sendo sete de pesquisa, dois de extensão e um de ensino, realizados pelos departamentos e programa, no período de 2010 a 2015. As informações coletadas foram analisadas com estatística descritiva, no programa SPSS v. 23.0. Resultados: constatou-se que a disseminação da produção do PPGPS ocorre, principalmente, através dos artigos científicos $(n=283)$, informativos $(n=11)$, vídeos documentários $(n=9)$ e internet (site ou blog do programa) $(n=17)$. Além disso, para cada projeto realizado pelo programa foi produzido, pelo menos, um documentário. Considerações finais: o PPGPS contou com produtos próprios, destinados a divulgar notícias, informações e produções científicas de projetos de mestrandos e professores do Programa. Identificou-se que o PPGPS faz uso de uma ampla variedade de recursos comunicacionais que possibilitam a socialização do conhecimento produzido pelo programa para um espectro maior, em rede de comunicação. Assim, a comunicação se estabelece em variados contextos sociais e geográficos próximos e distantes, oferecendo contribuições para educação continuada dos profissionais de saúde e professores da educação básica, possibilitando reflexões e dispositivos para a atuação profissional.
Palavras-chave: Promoção da Saúde; Interdisciplinaridade; Pesquisa; Extensão; Comunicação Social.

\section{ABSTRACT}

Objective: describe the use of social communication resources for teachers, researchers and postgraduate students of the Health Promotion Post-Graduation Program (PPGPS) of University of Santa Cruz do Sul. Method: for data collection, eleven informative were reviewed and a portion of the projects (10), seven of research, two extension and one education made by departments and Post-graduation program from 2010 to 2015. The data were analyzed with descriptive statistics, using SPSS program v. 23.0. Results: it was found that the PPGPS production spread occurs mainly through scientific articles ( $n=283$ ), informative ( $n=$ 11), documentary videos ( $n=9$ ) and internet (site or program blog) ( $n=17$ ). In addition, for each project carried out by the program is produced at least one documentary. Closing remarks: the PPGPS counted on own products, to disseminate news, information and scientific works of students and teachers projects. It was identified that the PPGPS makes use of a wide variety of communication features that enable the socialization of the knowledge produced by the program to a wider range in network communication. So communication is established in various social and geographic contexts near and far, providing contributions to continuing education of health professionals and basic education teachers, enabling reflections and devices for professional activities.

Key words: Health Promotion; Isciplinarity; Research; Extension; Social Communication 


\section{INTRODUÇÃO}

As mudanças propiciadas pelos avanços dos recursos tecnológicos e de mídias permitiram novas abordagens no meio acadêmico. ${ }^{1}$ No campo de pesquisa e extensão, esse avanço vem sendo marcado, especialmente, pela inserção dos recursos da Comunicação Social. ${ }^{2}$ O uso dos recursos de mídias visuais e de criação de documentários vem, de forma crescente, se caracterizando como ferramenta de disseminação do conhecimento científico, a fim de possibilitar aos docentes da universidade e comunidade acadêmica manterem-se atualizados e divulgarem, de forma mais efetiva, as suas atividades de pesquisa e ensino. ${ }^{3}$

Nesta perspectiva, a produção de conhecimento em saúde ampliou-se nos últimos anos em todas as suas esferas e tem adquirido novos significados. A integração dos recursos tecnológicos à produção de conhecimento fez emergir políticas científicas, as quais se apoiam sob a ótica de que tais produções devem contribuir para a melhor qualidade do atendimento em saúde, direcionando seu foco à socialização do conhecimento, bem como às necessidades de saúde das populações. ${ }^{4}$

Assim, é reconhecido que, para alcançar esses resultados, se faz necessária a implantação de ações que promovam a integração entre produção científica e os diversos setores sociais. . $^{45}$ A relevância de uma universidade, atualmente, passa a ser medida pela articulação que esta promove entre os três setores da prática acadêmica que a constitui: ensino regular e formal (formação acadêmica para atender às demandas sociais), pesquisa (investigação do conhecimento de forma metódica) e extensão - difusão (socialização do conhecimento junto à sociedade, visando criar referências às práticas sociais e laborais). ${ }^{6}$ Esta simbiose enriquece, dinamiza e gera mais motivação para a aprendizagem, quando se trata do ensino acadêmico, gerador e propulsor do desenvolvimento humano e da sociedade moderna. Deste modo, há um crescente incentivo ao desenvolvimento das ações de pesquisa e extensão e sugere-se que a integração de diferentes modalidades comunicacionais, como a escrita, oral e audiovisual, pode trazer grandes benefícios à concretização destas ações, no âmbito da saúde $^{5}$ e educação básica. ${ }^{7}$

Neste sentido, a Comunicação Social integrada à pesquisa, extensão e ensino exerce um papel fundamental; à medida que possibilita a cooperação e aproximação entre os pesquisadores, colabora para o reconhecimento das pesquisas e revalida as competências e a credibilidade do pesquisador, diante a comunidade científica. ${ }^{8}$ Ainda, a comunicação possui papel imprescindível no processo de democratização da ciência, da pesquisa e extensão, pois garante reconhecimento e legitimação, que irão assegurar apoio e recursos financeiros aos pesquisadores, e contribui no exercício crítico das políticas públicas de ciência, tecnologia e inovação. ${ }^{7-9} \mathrm{O}$ ensino, aliado à Comunicação Social, tornar-se-á mais atraente, atualizado e rico em sentidos, quando trouxer ao ambiente da formação acadêmica, a produção realizada no campo da pesquisa e extensão, principalmente se for vinculada, além de periódicos científicos, através de informativos, documentários, rede sociais, entre outros.

As possibilidades desses novos meios comunicacionais no campo acadêmico ainda estão em construção. ${ }^{10}$ Desta forma, considera-se a importância de conhecer, em diferentes perspectivas e com maior detalhamento, a implementação e gestão dos recursos da Comunicação Social na produção do conhecimento. Este trabalho tem por objetivo descrever o uso dos recursos de comunicação pelos docentes, pesquisadores e alunos do programa de Pós-Graduação em Promoção da Saúde (PPGPS) e de graduação, da Universidade de Santa Cruz do Sul (UNISC), nas atividades de pesquisa, ensino e extensão, voltadas à saúde na comunidade e na educação básica.

\section{MÉTODO}

A metodologia deste estudo descritivo (registro documental) consistiu de uma estratégia de exposição dinâmica da produção diversificada dos docentes, pesquisadores, mestres e mestrandos do Programa de Pós-Graduação em Promoção da Saúde (PPGPS), da Universidade de Santa Cruz do Sul (UNISC), com utilização de recursos de comunicação, que divulgam eventos, produtos e inovações tecnológicas, frutos das atividades de pesquisa e extensão voltadas à saúde na comunidade e educação básica.

A coleta de dados foi realizada de janeiro de 2010 a dezembro de 2015, com uma revisão de mídias produzidas - recursos de comunicação (tabela 1) e locais de divulgação (tabela 2) de partes dos dez projetos de pesquisa, de extensão e de ensino realizados pelo programa e departamentos envolvidos, relacionados também aos projetos de pesquisa e extensão dos orientadores e orientados. Por hora, foram focados projetos de pesquisa do programa que envolvem vários orientadores. Os projetos descritos no quadro 1 foram relacionados em razão de terem envolvimento de vários professores do PPGPS (como orientadores que via de regra tem também como coorientadores ou colaboradores outros professores do programa). Outras dissertações, em fase de conclusão serão defendidas em anos vindouros. As informações coletadas foram organizadas em tabelas e analisadas através do Programa SPSS v. 23.0 (IBM, Armonk, NY, USA), utilizando-se de frequência e percentual.

Os projetos são apresentados de forma sintética e, na sequência, é feita indicação de um ou mais links que remetem a informativos ou documentários ilustrativos das pesquisas e extensões realizadas.

\section{RESULTADOS}

Na composição desse estudo, foram analisados 11 informativos (cinco de 2014 e seis de 2015) e dez projetos de pesquisa, extensão e ensino, no período de 2010 a 2015. Estes projetos têm como foco divulgar, acompanhar e avaliar aspectos relacionados a ações ligadas ao Sistema Único de Saúde, a questões epidemiológicas, à saúde dos escolares da Educação Básica, à Saúde do trabalhador, à Vigilância da Saúde, bem como propor ações de intervenção como, por exemplo, de projeto de ginástica laboral, em unidade hospitalar. De acordo com os dados apresentados na tabela 1, é possível observar que a maior parte dos resultados, no caso deste estudo, de parte das pesquisas realizadas pelo PPGPS são divulgados, através dos artigos científicos 
Tabela 1 - Recursos de comunicação produzidos pelos PPGPS para divulgação das produções científicas.

\begin{tabular}{lccccccc}
\hline Material Produzido & $\mathbf{2 0 1 0}$ & $\mathbf{2 0 1 1}$ & $\mathbf{2 0 1 2}$ & $\mathbf{2 0 1 3}$ & $\mathbf{2 0 1 4}$ & $\mathbf{2 0 1 5}$ & Total \\
& $\mathbf{n}(\%)$ & $\mathbf{n}(\%)$ & $\mathbf{n}(\%)$ & $\mathbf{n}(\%)$ & $\mathbf{n}(\%)$ & $\mathbf{n}(\%)$ & $\mathbf{n}(\%)$ \\
\hline Vídeos documentários & $0(-)$ & $0(-)$ & $05(35,71)$ & $03(21,42)$ & $03(21,42)$ & $03(21,42)$ & $14(4,49)$ \\
Informativos & $0(-)$ & $0(-)$ & $0(-)$ & $0(-)$ & $05(45,45)$ & $06(54,54)$ & $11(3,53)$ \\
E-books & $0(-)$ & $0(-)$ & $0(-)$ & $0(-)$ & $01(50,00)$ & $01(50,00)$ & $02(0,64)$ \\
Artigos científicos & $36(12,72)$ & $37(13,07)$ & $59(20,84)$ & $65(22,96)$ & $53(18,72)$ & $34(12,01)$ & $283(90,70)$ \\
Folderes & $0(-)$ & $01(50,00)$ & $01(50,00)$ & $0(-)$ & $0(-)$ & $0(-)$ & $02(0,64)$ \\
\hline
\end{tabular}

$(n=283)$, sendo a divulgação realizada por periódicos científicos, informativos e vídeos documentários. Além disso, os dados da tabela apontam que a quantidade de publicações e produtos produzidos pelo programa, que vem crescendo a cada ano. Ressalta-se que em 2015, houve uma queda numérica de artigos, porém houve uma progressão na qualidade dos artigos.

A tabela 2 mostra os locais de divulgação das produções científicas, sendo que os principais meios em que as produções foram divulgadas, são publicações em revistas científicas $(n=283$ ) e internet (site ou blog do programa) $(n=22)$.

Tabela 2 - Locais de divulgação das produções científicas produzidas.

\begin{tabular}{lc}
\hline Locais de divulgação & $\mathbf{n}(\%)$ \\
\hline Publicações em revistas científicas & $283(92,18)$ \\
Internet (site ou blog do programa) & $22(7,17)$ \\
Exposição na instituição & $2(0,65)$ \\
\hline
\end{tabular}

*tabela de alternativas múltiplas
Alguns dos projetos de pesquisa, dos quais originaram dissertações do programa, ao longo dos últimos anos, estão descritos no quadro 1, com seus respectivos orientadores e mestrandos, bem como dois Pós Doc, que integraram seus trabalhos ao projeto Casadinho. As dissertações podem ser encontradas no portal da CAPES, bem como na página do Programa, na íntegra. Link para a página do PPGPS:

http://www.unisc.br/portal/pt/cursos/mestrado/mestrado-em-promocao-da-saude/apresentacao.htm/

$\mathrm{Na}$ sequência, apresentaremos o Informativo PPGPS, a descrição sintética dos projetos de pesquisa, extensão, ensino e e-books que compõem a amostra deste estudo. Aos projetos, seguem-se links de documentários produzidos, via de regra, de curta duração, ou em alguns casos, mais longos, detalhando palestras e cursos de transferência de tecnologia à populações (exemplo: agricultores da agro-indústria, seus filhos, escolares da educação básica, usuários de projetos de extensão da UNISC, do HSC - Hospital Santa Cruz, entre outros).

Quadro 1 - Exemplificação de dissertações defendidas por projetos hospedeiros de pesquisa e orientador.

\begin{tabular}{|c|c|c|c|c|c|c|}
\hline \multirow[t]{2}{*}{ Titulo do Projeto } & \multirow{2}{*}{$\begin{array}{l}\text { Orientador e } \\
\text { Coorientador }\end{array}$} & \multicolumn{4}{|c|}{ Orientandos PPGPS e Pós-Doc } & \multirow[b]{2}{*}{ Total } \\
\hline & & 2012 & 2013 & 2014 & 2015 & \\
\hline $\begin{array}{l}\text { AVALIAC̣ÃO DE INDICADORES BIO- } \\
\text { QUíMICOS DE SAÚDE DE ESCOLARES } \\
\text { USANDO ESPECTROSCOPIA NO } \\
\text { INFRAVERMELHO: um estudo em Santa } \\
\text { Cruz do Sul- FASE I 2010-2011 }\end{array}$ & $\begin{array}{l}\text { Valeriano Antonio } \\
\text { Corbellini }\end{array}$ & - & - & - & $\begin{array}{c}\text { Francielle Pasqualotti } \\
\text { Meinhardt } \\
\text { http://www.unisc.br/portal/ } \\
\text { upload/com_arquivo/fran- } \\
\text { cielle_meinhardt.pdf }\end{array}$ & 1 \\
\hline \multirow{5}{*}{$\begin{array}{l}\text { OBESIDADE E COMORBIDADES EM } \\
\text { ESCOLARES E SUA RELAC̣ÃO COM } \\
\text { FATORES DE RISCO ÀS DOENC̣AS } \\
\text { CRÔNICAS E INFECCIOSAS: uma } \\
\text { abordagem interdisciplinar } \\
\text { Projeto CASADINHO } \\
2012-2015\end{array}$} & $\begin{array}{l}\text { Miria Suzana Burgos } \\
\text { Andréia Rosane de } \\
\text { Moura Valim }\end{array}$ & - & $\begin{array}{l}\text { Cézane Priscila Reuter } \\
\text { http://www.unisc.br/ } \\
\text { portal/upload/com_ar- } \\
\text { quivo/1435866768.pdf }\end{array}$ & - & - & \multirow{3}{*}{3} \\
\hline & $\begin{array}{l}\text { Andréia Rosane de } \\
\text { Moura Valim } \\
\text { Lia Gonçalves Possuelo }\end{array}$ & - & - & $\begin{array}{l}\text { Pâmela Ferreira Todendi } \\
\text { http://www.unisc.br/por- } \\
\text { tal/upload/com_arquivo/ } \\
\text { dissertacao_pamela.pdf }\end{array}$ & - & \\
\hline & $\begin{array}{c}\text { Andréia Rosane de } \\
\text { Moura Valim }\end{array}$ & - & - & Lisiane Lisboa & - & \\
\hline & $\begin{array}{l}\text { Marilene Henning } \\
\text { Vaistein (UFRGS) }\end{array}$ & - & - & - & $\begin{array}{c}\text { Pós-doc Prof. Dr. Jorge } \\
\text { Horta }\end{array}$ & \\
\hline & $\begin{array}{l}\text { Ionara Pizzutti } \\
\text { (UFSM) }\end{array}$ & - & - & - & $\begin{array}{c}\text { Pós-doc Prof. Dr. Valeriano } \\
\text { Antônio Coberllini }\end{array}$ & \\
\hline \multirow{3}{*}{$\begin{array}{l}\text { AVALIAC̣ÃO DE INDICADORES } \\
\text { BIOQUÍMICOS DE SAÚDE DE ESCO- } \\
\text { LARES USANDO ESPECTROSCOPIA } \\
\text { NO INFRAVERMELHO, POLIMORFIS- } \\
\text { MOS, SAÚDE BUCAL E FATORES } \\
\text { RELACIONADOS AO ESTILO DE VIDA: } \\
\text { um estudo em Santa Cruz do Sul - } \\
\text { FASE II 2012-2013 }\end{array}$} & Miria Suzana Burgos & - & - & $\begin{array}{l}\text { Tássia Silvana Borges } \\
\text { http://www.unisc.br/por- } \\
\text { tal/upload/com_arquivo/ } \\
\text { dissertacao_tassia_1.pdf }\end{array}$ & - & \multirow{3}{*}{3} \\
\hline & Lia Gonçalves Possuelo & - & - & - & Cassiano Macari & \\
\hline & $\begin{array}{l}\text { Daniel Prá } \\
\text { Silvia Isabel Rech } \\
\text { Franke }\end{array}$ & - & - & - & $\begin{array}{c}\text { Karini da Rosa } \\
\text { http://www.unisc.br/portal/ } \\
\text { upload/com_arquivo/ka- } \\
\text { rini_da_rosa.pdf }\end{array}$ & \\
\hline
\end{tabular}




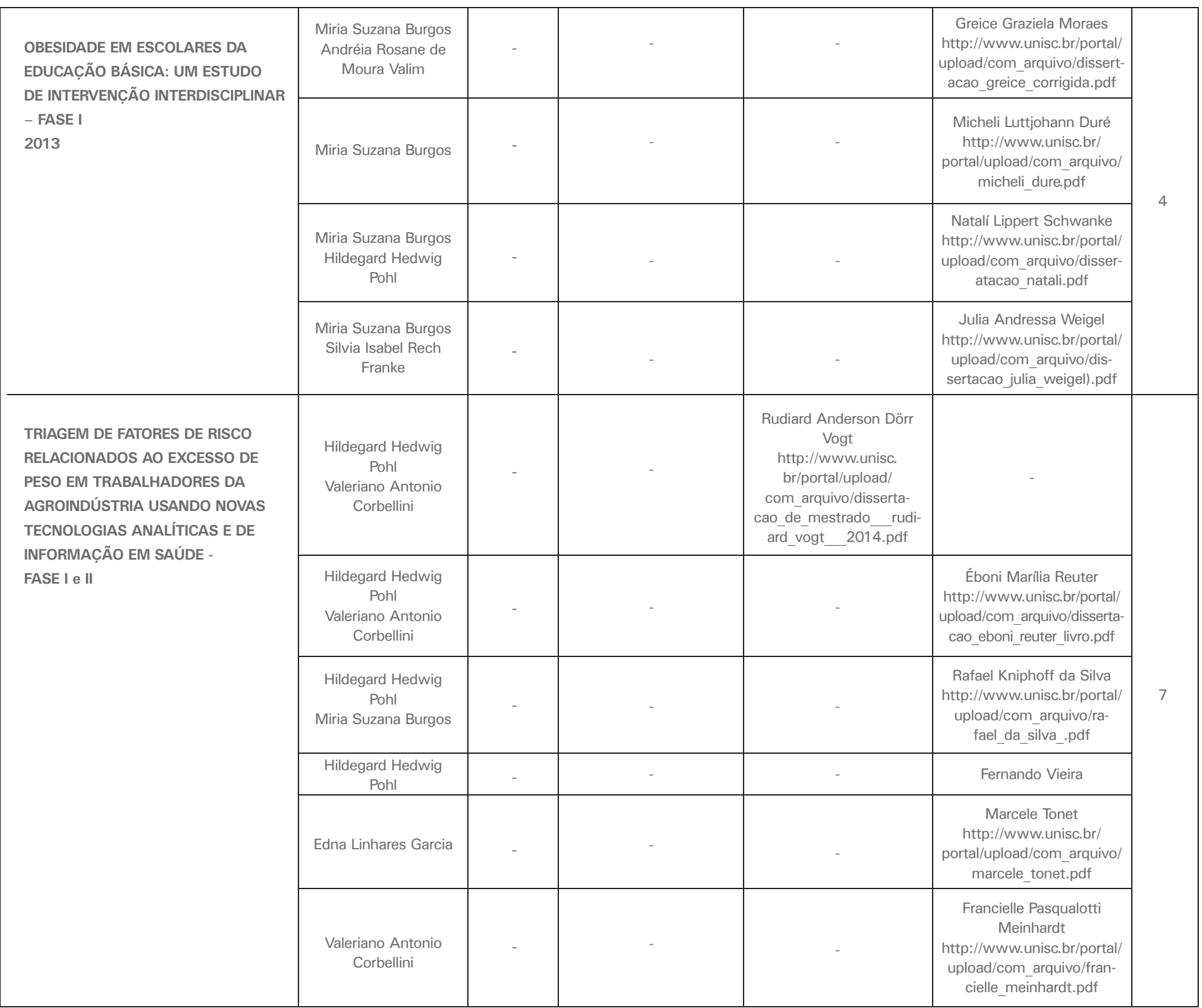

\section{INFORMATIVOS}

Informativo PPGPS: faz parte do projeto de Pesquisa e Extensão EXTPEQ. Foram realizadas seis edições mensais no segundo semestre de 2014 e seis bimestrais no ano de 2015. Nas edições, constam informações acerca de projetos de pesquisa e extensão desenvolvidos pelos mestrandos e docentes do PPGPS, momentos especiais nas atividades de ensino, quando parceiros da comunidade e da academia são convidados a participar da construção do conhecimento que se dá na relação da sala de aula. Também foram abordadas notícias referentes à promoção e participação em eventos científicos, com o objetivo de disseminar o conhecimento produzido pelos alunos e docentes, promovendo maior visibilidade das atividades desenvolvidas no PPGPS. Destacamos a edição 6 - ano II - Novembro e Dezembro de 2015 - que relata o II Seminário Científico. Para visualização dos Informativos, dispomos os links.
Links para os informativos de 2014:

- Informativo PPGPS - Ed. 1 - Ano I - Agosto: http://unisc.br/portal/upload/com _arquivo/informativo _ mestrado_agosto.jpg

- Informativo PPGPS - Ed. 2 - Ano I - Setembro: http://unisc.br/portal/upload/com _arquivo/informativo _ mestrado_setembro.jpg

- Informativo PPGPS - Ed. 3 - Ano I - Outubro: http://unisc.br/portal/upload/com _arquivo/informativo _ mestrado_outubro.jpg

- Informativo PPGPS - Ed. 4 - Ano I - Novembro: http://unisc.br/portal/upload/com _arquivo/informativo _mestrado _novembro.jpg _ 2.jpg

- Informativo PPGPS - Ed. 5 - Ano I - Dezembro: http://unisc.br/portal/upload/com _arquivo/informativo _mestrado _dezembro _(2).jpg 
Links para os informativos de 2015:

- Informativo PPGPS - Ed. 1 - Ano II - Janeiro e Fevereiro: http://issuu.com/ppgps/docs/informativo ppgps $2015_{-}{ }_{-}$edi ${ }_{-}{ }_{-} o_{-} 1$

- Informativo PPGPS - Ed. 2 - Ano II - Março e Abril: http://issuu.com/ppgps/docs/informativo ppgps $2015_{-}{ }_{-}{ }_{-} e d i{ }_{-}{ }_{-} o_{-}$

- Informativo PPGPS - Ed. 3 - Ano II - Maio e Junho: ht $t p: / / i s s u$. co $\mathrm{m} / \mathrm{ppg} \mathrm{ps} / \mathrm{doc} s / i n$ formativo_ppgps_2015 _ _ edi $\quad o \quad$ 8585fe4735e79̄8

- Informativo PPGPS - Edição 4 - Ano II - Julho e Agosto:

ht t p://issuu.com/ppgps/docs/informativo_ppgps _ $2015 \ldots \ldots$ edi__ _ $o_{-} 1 \overline{6} 24326 a 24 e 044$

- Informativo PPGPS - Ed. 5 - Ano II - Setembro e Outubro: ht t $p: / / i s s u$ i c com/ppgps/docs/in. formativo_ppgps $2015 \ldots \ldots$ edi_ _ _ o _ $30 e 2 b d 4241 b 333$

- Informativo PPGPS - Ed. 6 - Ano II - Novembro e Dezembro: http://issuu.com/ppgps/docs/informativo ppgps $2015_{-}{ }_{-}{ }_{-}$edi _ _ _ ${ }_{-} o_{-}$f010a43dbbe7e6

\section{PROJETOS DE PESOQUISAS:}

A pesquisa "AVALIAÇÃO DE INDICADORES BIOQUÍMICOS DE SAÚDE DE ESCOLARES USANDO ESPECTROSCOPIA NO INFRAVERMELHO: um estudo em Santa Cruz do Sul - Fase I - 2010-2011" objetiva investigar metodologia analítica, à base de espectroscopia no infravermelho para quantificar marcadores bioquímicos em sangue, plasma e soro, visando a avaliação precoce de riscos a patologias de escolares da zona rural e urbana de Santa Cruz do Sul. O estudo piloto (2010) e de amostra populacional (2011), do tipo transversal, com base em dados quantitativos, tiveram uma amostra de 50 sujeitos, retirada da amostra (de pesquisa realizada de 2007 a 2009) de 1578 escolares, do Ensino Fundamental e Médio, da rede pública e privada do município de Santa Cruz do Sul, com idade entre 7 e 17 anos, abrangendo a zona urbana e rural. Os principais procedimentos metodológicos foram: $1^{\circ}$ ) capacitação sobre fundamentos teórico-práticos dos procedimentos experimentais do projeto, organização e padronização de procedimentos de métodos e técnicas de avaliação; $2^{\circ}$ ) aplicação de duas etapas de testagem: a) etapa de calibração para criar o protocolo, com uma amostra de 20 a 30 indivíduos e b) etapa de validação do protocolo, para verificar a confiabilidade do protocolo de calibração, com outra amostra de 20 a 30 indivíduos, com características semelhantes das do grupo que participou da etapa de calibração. $3^{\circ}$ ) aplicação do protocolo validado, para triagem de dados bioquímicos que se correlacionam com prevenção de doenças crônico-degenerativas de escolares da zona urbana e rural de Santa Cruz do Sul (isto, na continuação do projeto em 2011).
A pesquisa "AVALIAC̣ÃO DE INDICADORES BIOQUÍMICOS DE SAÚDE DE ESCOLARES USANDO ESPECTROSCOPIA NO INFRAVERMELHO, POLIMORFISMOS, SAÚDE BUCAL E FATORES RELACIONADOS AO ESTILO DE VIDA: um estudo em Santa Cruz do Sul - Fase II - 2012-2013" investigou metodologia analítica, à base de espectroscopia no infravermelho, para quantificar marcadores bioquímicos em sangue, plasma e soro, visando à avaliação precoce de riscos a patologias de escolares da zona rural e urbana de Santa Cruz do Sul. Investigou polimorfismos genéticos, problemas cardiovasculares, fatores de risco às doenças cardiovasculares, desempenho somatomotor, estilo de vida e saúde bucal. A amostra populacional comportou um total aproximado de 2000 sujeitos, escolares de Santa Cruz do Sul, do Ensino Fundamental e Médio, da rede pública e privada, com idade entre 7 e 17 anos, abrangendo a zona urbana e rural. Os principais procedimentos metodológicos foram: $1^{\circ}$ ) capacitação sobre fundamentos teórico-práticos dos procedimentos experimentais do projeto, organização e padronização de procedimentos de métodos e técnicas de avaliação; $2^{\circ}$ ) triagem de dados bioquímicos, a partir da coleta de sangue, que se correlacionam com prevenção de doenças crônico-degenerativas de escolares da zona urbana e rural de Santa Cruz do Sul; $3^{\circ}$ ) avaliação de polimorfismos genéticos, fatores de risco às doenças cardiovasculares (obesidade e hipertensão arterial), desempenho somatomotor, indicadores de saúde (IMC, flexibilidade, força abdominal e capacidade cardiorrespiratória) e estilo de vida; $4^{\circ}$ ) avaliação da saúde bucal, com protocolo específico da OMS, prevalência de cárie dentária e necessidade de tratamento.

A pesquisa "SAÚDE DOS ESCOLARES: AVALIAÇÃO DE INDICADORES BIOQUÍMICOS, GENÉTICOS, HEMATOLÓGICOS, IMUNOLÓGICOS, POSTURAIS, SOMATOMOTORES, SAÚDE BUCAL, FATORES DE RISCO ÀS DOENCAS CARDIOVASCULARES E ESTILO DE VIDA DE ESCOLARES: um estudo em Santa Cruz do Sul-RS - Fase III - 2014-2015" objetivou investigar a saúde dos escolares, visando diagnóstico precoce de desenvolvimento de possíveis patologias. As coletas de dados foram realizadas em 25 escolas do município, com amostra de aproximadamente 2000 escolares de 7 a 17 anos. As avaliações foram realizadas no complexo esportivo da UNISC, e envolveram a realização de antropometria, aferição da pressão arterial, exames de sangue, urina e fezes, avaliação da frequência cardíaca de repouso e de esforço, teste de maturação sexual e percepção/satisfação corporal, aplicação de questionário sobre o estilo de vida e avaliação da aptidão física. Os alunos participantes receberam laudos dos exames, posteriormente, na escola. Link para documentário: https://www.youtube.com/watch?v=Wv3Mir1u16E

A pesquisa "OBESIDADE EM ESCOLARES DA EDUCAC̣ÃO BÁSICA: UM ESTUDO DE INTERVENÇÃO INTERDISCIPLINAR - FASE I" foi desenvolvida no período de agosto a novembro de 2013 , com crianças e adolescentes de 8 a 16 anos diagnosticados com sobrepeso e obesidade, em uma escola municipal do meio rural de Santa Cruz do Sul. As atividades propos- 
tas envolveram professores e bolsistas do PPGPS e da graduação; incluíram exercícios físicos programados, reeducação postural, orientação nutricional e orientação de saúde bucal. Link para documentários:

a) Orientação postural:

https://www.youtube.com/watch? $v=m G q u 8 U t-p V s$

b) Atividade física orientada - aula:

https://www.youtube.com/watch?v=G5AQDGcvsd8

c) Orientação nutricional:

https://www.youtube.com/watch?v= ${ }_{5} 5 \mathrm{JpIRxvUZO}$

A pesquisa "OBESIDADE EM ESCOLARES DA EDUCAÇÃO BÁSICA: UM ESTUDO DE INTERVENÇÃO INTERDISCIPLINAR - FASE II" foi desenvolvida no período de abril a setembro de 2015, com adolescentes de 11 a 17 anos diagnosticados com sobrepeso e obesidade. As atividades propostas envolveram bolsistas do PPGPS e da graduação, incluindo exercícios físicos programados e orientados por profissionais de Educação Física três vezes por semana, orientação psicológica, por psicólogo e orientação nutricional por nutricionistas e bolsistas uma vez por semana, visando uma melhora no estilo de vida e na saúde dos participantes. Trabalharam na intervenção: no grupo de nutrição, a mestre Patrícia Molz, a mestre e doutoranda Fabiana Assmann Poll e bolsistas da graduação: Luana Beatriz Limberger, Caroline dos Santos, Diene da Silva Schlickmann, Fernanda da Silva Pfeifer e Camila dos Santos; no grupo de Educação Física, os mestrandos Carlos Ferreira Hoehr, Débora Tornquist, Léticia Borfe, Luciana Tornquist, Luiza Naurjorks Reis e Tauana Arcadepani Silvério e bolsistas da graduação: Cleiton Bertollo, Deise Graziela Kern, Ana Paula Sehn, Priscila Tatiana da Silva, Marcus Daniel Silva Camargo e Camila Billing. E da psicologia, o mestrando Lucas Lüdtke. Link para documentários:

a) Orientação nutricional (oficinas e sessões de estudo): https://www.youtube.com/watch? $v=T L c V D B J c k V c$

b) Orientação de atividades físicas para redução de peso: https://www.youtube.com/watch?v=ZtObGAhCoCg

A pesquisa "Casadinho" (financiamento Capes/ CNPq), intitulada "OBESIDADE E COMORBIDADES EM ESCOLARES E SUA RELAÇÃO COM FATORES DE RISCO ÀS DOENÇAS CRÔNICAS E INFECCIOSAS: uma abordagem interdisciplinar" tem o objetivo de fortalecer e consolidar o Programa de Pós-Graduação em Promoção da Saúde da Universidade de Santa Cruz do Sul (UNISC), pela parceria com o Programa de Pós- Graduação em Biologia Celular e Molecular (PPGBCM) da Universidade Federal do Rio Grande do Sul (UFRGS). A proposta de pesquisa apresentada visa abordar a obesidade e comorbidades em escolares e sua relação com fatores de risco às doenças crônicas e infecciosas, através de uma abordagem interdisciplinar. Link para documentário:

https://www.youtube.com/watch?v=YTfCCPS703A

A pesquisa "Triagem de fatores de risco relacionados ao excesso de peso em trabalhadores da agroindústria usando novas tecnologias analíticas e de informação em saúde - Fase I e II - 2013-2015", orientada pela professora Hildegard Hedwig Pohl, visa fazer a triagem dos trabalhadores de agroindústria dos municípios de Santa Cruz do Sul (cidade polo do Conselho Regional de Desenvolvimento do Vale do Rio Pardo-COREDE/ VRP), Vale Verde, Passo do Sobrado, Encruzilhada do Sul, Candelária, Pantano Grande, Rio Pardo e General Câmara, identificando os possíveis fatores de risco relacionados ao excesso de peso. A população selecionada está alocada nos municípios que compõem a Microrregião Sul do COREDE do Vale do Rio Pardo, escolhida em virtude de ser uma região carente de assistência no âmbito social e da saúde. A avaliação destes trabalhadores enfocou a aptidão metabólica através de marcadores bioquímicos, antropométricos e fisiológicos, buscando desenvolver metodologia à base da espectroscopia do infravermelho com Transformada de Fourier (FT-IR) através de avaliação global. Esta avaliação constou de dois momentos: a) aptidão metabólica crônica em jejum segundo exames bioquímicos de rotina para triagem de fatores de risco associados ao excesso de peso e b) aptidão metabólica aguda em estado pós-absortivo simultânea à avaliação antropométrica, bioquímica e da aptidão funcional cardiorrespiratória, que além dos métodos tradicionais foram analisados por espectroscopia no infravermelho associada a métodos de análise multivariada visando uma única análise espectroscópica (avaliação global). As avaliações ocorreram no primeiro momento em forma de triagem e no segundo momento como forma de acompanhamento e monitorização dos fatores de risco encontrados como os riscos de doenças cardiovasculares, a presença de pré-diabéticos e as alterações posturais constatadas na primeira fase da pesquisa. Para além das orientações e recomendações de cuidados com a saúde aos trabalhadores, propõe-se também instrumentalizar os órgãos de saúde dos municípios, divulgando os dados e desenvolvendo estratégias de promoção da saúde. Link para documentário: https://www.youtube.com/watch?v=xEfvOMOg6/c

\section{PROJETO DE ENSINO - PIBID}

O Programa Institucional de Bolsas de Iniciação à Docência (PIBID - UNISC), parte do Programa Nacional de Iniciação à Docência PIBID - CAPES, consiste em uma política pública com finalidade de aperfeiçoar a formação de professores e apoiar a iniciação à docência de estudantes de licenciaturas das universidades brasileiras com ações voltadas à educação básica. Para isso, o programa oferece bolsas de estudos para estudantes das diversas áreas dos cursos das licenciaturas e também para os professores das escolas públicas que participam do programa. O objetivo principal do PIBID é articular a vivência cotidiana dos licenciandos com a educação básica, propondo a convivência de forma contínua, com toda a complexidade que se materializa na escola. Considerando que, através da inserção qualificada na escola, os estudantes de licenciaturas poderão articular elementos de ensino/pesquisa, teoria/ prática e ação/reflexão. No caso do presente relato, o subprojeto de Educação Física (único curso da Saúde que possui licenciatura), da UNISC, coordenado pelas professores Miria Suzana Burgos e Miriam Beatris Reckziegel, visa possibilitar aos acadêmicos a vivência de metodologias, recursos e estratégias educacionais, 
resolvendo problemas ou sugerindo ações para aprimorar o aproveitamento da contribuição da ciência para a comunidade escolar. Neste sentido, os acadêmicos do PIBID participantes do subprojeto de Educação Física, juntamente com as atividades de iniciação à docência, tem também a oportunidade de participar das atividades de coleta de dados das pesquisas de saúde dos escolares, enriquecendo de forma mais ampliada a formação dos acadêmicos na integração do ensino com pesquisa interdisciplinar do PPGPS, capacitando-os à avaliação de escolas em dimensões culturais do estilo de vida, hábitos nutricionais, práticas de atividade física, indicadores de saúde, desenvolvimento motor e fatores de risco às doenças cardiovasculares relacionados à saúde do escolar, que envolve todas as áreas de saúde. Link para documentário:

https://www.youtube.com/watch?v=tkfTjfbQpjg

\section{PROJETO DE EXTENSÃO}

O projeto de extensão "GINÁSTICA LABORAL E ATIVIDADES DE INTERVENÇÃO DO PROFISSIONAL DA EDUCAÇÃO FÍSICA NO HSC (Hospital Santa Cruz) - Fase I - 2014 e Fase II - 2015" tem por objetivo implementar a Ginástica Laboral (GL) no HSC e, através desta, promover adaptações fisiológicas, físicas e psíquicas nos sujeitos participantes por meio de exercícios dirigidos. Além disto, busca acompanhar a realização de atividades físicas e recreativas hospitalares junto a crianças, adolescentes e adultos, pacientes e/ou funcionários do HSC, buscando promover a saúde e bem estar. O projeto também está comprometido com o desenvolvimento de ações que envolvam ensino e pesquisa; assim, acompanha as atividades de intervenção curriculares dos alunos do curso de Educação Física da UNISC e demais cursos da área da saúde envolvidos no Mestrado em Promoção da Saúde da UNISC e Residência Multiprofissional na Saúde do Hospital Santa Cruz. Para estes, os procedimentos, atividades, protocolos de coleta de dados adotados e respectivos cronogramas seguem o estabelecido nos respectivos projetos de pesquisa, aprovados pelo Comitê de Ética. Está em processo de finalização, uma dissertação de mestrado, a ser defendida em fevereiro-2016, razão pela qual não foi contabilizada neste estudo. Link para documentário: https://www.youtube.com/watch?v=9nC6RSXnJyY

O projeto de extensão EXTPEQ - EXTENSÃO E PESQUISA - SOCIALIZAC̣ÃO DA PRODUÇÃO DO CONHECIMENTO: SAÚDE-EDUCAÇÃO-TRABALHO - FASE II - 2015 tem como objetivo promover a disseminação regional e internacionalizar o conhecimento produzido no Mestrado em Promoção da Saúde, associado às pesquisas e extensões dos docentes e alunos do PPGPS, bem como dos demais departamentos e cursos da UNISC relacionados à saúde. Algumas ações previstas para o projeto envolvem a publicação das mídias e produtos técnicos desenvolvidos em eventos regionais, nacionais e internacionais no site do PPGPS, a fim de contribuir no processo de esclarecimento e emancipação das populações locais e regionais, permitindo refletir, em outras esferas geográficas mais abrangentes, sobre promoção da saúde e qualidade de vida. Como estratégia metodológica, o projeto visa à adoção de diversos recursos, entre eles a utilização de encontros de capacitação dos envolvidos nos processos, filmagens, registros fotográficos, entrevistas, reportagens, notas à imprensa, uso de redes sociais - como Facebook -, mensagens eletrônicas e elaboração do Informativo PPGPS da UNISC. Todos esses aspectos visam trazer inovações científicas e tecnológicas para o campo de pesquisa em promoção da saúde e qualidade de vida. Deste projeto, foi originado o trabalho desenvolvido no presente artigo.

\section{LIVROS ELETRÔNICOS - e-books}

Tem-se destacado, a partir de 2014, a publicação de livros eletrônicos (e-books) com textos científicos com linguagem acessível ao público em geral, principalmente a professores da educação básica que lidam com crianças e jovens em desenvolvimento e a profissionais que lidam no Sistema Único de Saúde (SUS), unidades hospitalares e clínicas de atendimento à população em geral. Assim, professores e profissionais da saúde podem enriquecer seu aporte teórico que dê sustentação às práticas profissionais cotidianas, buscando melhorias na iniciação à docência e no sistema educacional

O primeiro e-book do PPGPS foi publicado em 2014, intitulado “Promoção da Saúde na Educação Básica: Interdisciplinaridade nos parâmetros curriculares, competências, habilidades e conteúdos" (Link para o e-book: http://www.unisc.br/portal/upload/com editora_livro/e_book05215.pdf

O segundo e-book- edição de 2015 - encontra-se em processo de finalização, sendo intitulado "Interdisciplinaridade e Promoção da Saúde na Educação Básica e no Sistema de Saúde", que poderá ser encontrado no site da EDUNISC (link para acesso a página da editora: http://www.unisc.br/portal/pt/editora/apresentacao.htm/

\section{DISCUSSÃO}

Geralmente, os resultados das produções científicas permanecem limitados aos meios em que são produzidas, sem alcançar abrangência e repercussão na sociedade. ${ }^{11}$ No entanto, nosso estudo constatou que a comunicação é ferramenta essencial para disseminação do conhecimento científico produzido pelo PPGPS. Desta forma, a comunicação possibilita socializar o conhecimento para um âmbito maior, como escolas, serviços de saúde e atingir esferas na web inimagináveis, cumprindo com sua missão de contribuir para melhorar a qualidade do atendimento em saúde, de acordo com as necessidades de saúde das populações, tanto no âmbito do sistema de saúde, quanto na educação básica.

Outro achado importante é que o PPGPS utiliza uma grande quantidade de recursos comunicacionais que incluem desde artigos científicos, elaboração de informativos e e-books. Desta forma, o conhecimento produzido pelo programa consegue, através da comunicação, englobar uma ampla gama de indivíduos em vários contextos sociais e geográficos. ${ }^{12}$ Isso se torna possível pois os meios de comunicação apresentam, nos dias atuais, a capacidade de diminuir as distâncias 
geográficas e as diferenças entre as linguagens técnico-científicas aplicadas pelos pesquisadores da área da saúde e o público. ${ }^{13}$ Assim, os recursos de comunicação, especialmente o Jornalismo científico, são vistos e inseridos pelo programa como instrumentos que permitem romper fronteiras e internacionalizar os avanços e achados científicos. ${ }^{14}$

Além disso, outro ponto positivo encontrado neste estudo diz respeito aos meios de comunicação em que as produções são divulgadas, com crescente divulgação através de publicações em revistas científicas $(n=283)$ e internet, site ou blog do programa $(n=17)$. Sabe-se que a mídia, através de diferentes mecanismos, como rádio, vídeos, documentos digitais e jornais, propicia a disseminação do conhecimento e estes podem ser utilizados para incentivar hábitos que visem à promoção da saúde e melhora da qualidade de vida da população. ${ }^{15}$ Nesse sentido, considerando que as pesquisas e projetos investigados nesses estudos abrangem aspectos referentes ao estilo de vida e indicadores de saúde de trabalhadores e escolares da educação básica, o uso dos recursos da comunicação se tornam amplamente relevantes por contribuir no compartilhamento e socialização de informações qualificadas e estimular o debate em torno de temas de saúde e educação.

Desta forma, a comunicação em saúde deve ser repensada constantemente visto que uma das principais aprendizagens que se pode destacar, a partir desse estudo, é que, apesar de a comunicação colaborar com a divulgação de informações científicas importantes, tanto no âmbito da saúde como na educação, deve-se ter especial cuidado com a forma de acessibilidade, foco e direção ao público interessado.

\section{CONSIDERACÕES FINAIS}

Neste estudo, identificou-se que o PPGPS faz uso de uma ampla variedade de recursos comunicacionais, sendo que o uso da Comunicação Social está focado em possibilitar a socialização do conhecimento produzido pelo programa para um âmbito maior, abrangendo indivíduos em vários contextos sociais e geográficos, como escolas e serviços de saúde, contribuindo de forma mais efetiva para melhora da qualidade do atendimento em saúde. Além disso, o PPGPS contou com produtos próprios de divulgação, como o Informativo PPGPS e documentários, ambos destinados a vincular notícias e informações acerca das pesquisas dos mestrandos e professores do Programa.

Assim, constata-se que a integração entre pesquisa, ensino, extensão e Comunicação Social se reflete na qualificação do ensino, permitindo ao programa uma alternativa diferenciada para produzir e divulgar o conhecimento científico, à medida que o conhecimento não esteve limitado apenas aos locais em que foram produzidos. $O$ artigo permitiu identificar também que o Programa, em todas as suas linhas de pesquisa, apresenta crescimento notável na quantidade de publicações científicas produzidas. Ressalta-se, deste modo, que a utilização dos meios de comunicação possibilitam à ciência alcançar maior notoriedade nas pesquisas desenvolvidas, informando a população e a comunidade científica sobre os resultados relevantes que são obtidos através das pequisas e atividades de ensino e extensão. Portanto, este novo contexto traz a criatividade e a inovação para a atuação interdisciplinar e contribui com ações mais efetivas no processo formativo em saúde e educação básica.

\section{PERSPECTIVAS:}

Para o ano de 2016 estaremos ampliando as fronteiras do presente estudo, em novo artigo, incluindo outros projetos do programa e incremento dos projetos já expostos, novas dissertações e produções científicas publicadas, entre outros. Nossa prioridade é o envolvimento interdisciplinar dos pesquisadores, mestrandos, mestres e bolsistas da graduação com diferentes formações, para que possam refletir e produzir com foco na promoção da saúde e bem estar da população.

\section{AGRADECIMENTOS}

Ao Programa de Bolsas de Extensão (PROBEX UNISC) pela bolsa de estudos concedida aos acadêmicos do curso de Comunicação Social - Habilitação em Jornalismo e Habilitação em Produção em Mídia Audiovisual - UNISC; e ao Programa de Suporte à Pós-Graduação de Instituições de Ensino Particulares (PROSUP - CAPESI pela bolsa de estudos concedida à Professora de Educação Física, Mestranda do Programa de Pós-Graduação em Promoção da Saúde - PPGPS/UNISC.

Os autores e o PPGPS agradecem às demais instituições pelos auxílios prestados ao longo das pesquisas descritas nesse estudo: CNPq, pelas bolsas PIBIC - CNPq e PIBITI CNPq; à FAPERGRS pelas bolsas PROBIC - FAPERGS e PROBITI - FAPERGS; à UNISC pelas bolsas PUIC - UNISC e PUIC - Inovação Tecnológica UNISC.

\section{REFERÊNCIAS}

1. Rodrigues C, Blattmann U. Gestão da informação e a importância do uso de fontes de informação para geração de conhecimento. Perspectivas em Ciência da Informação 2014;19(3):4-29.

2. Romaniello MM, Guimarães PTG. Interação pesquisa-extensão: uma análise da comunicação entre os atores sociais no processo de difusão e transferência de inovações tecnológicas para o agronegócio café. Interface 2008;5(2):80-96.

3. Cuenca AMB, Tanaka ACD. As novas tecnologias na comunicação científica: o uso da Internet pela comunidade de docentes da área de saúde pública. In: Anais Do Encontro Nacional de Pesquisa em Ciência da Informação, 5, 2003, Belo Horizonte. Anais. Belo Horizonte: UFMG, 2003.

4. Novaes HMD, Elias FTS. Uso da avaliação de tecnologias em saúde em processos de análise para incorporação de tecnologias no Sistema Único de Saúde no Ministério da Saúde. Cad Saude Publica 2013;29(1):7-16. DOI: http:// dx.doi.org/10.1590/0102-311X00008413.

5. Oliveira VC. Media communication and the Single Healthcare System. Interface Comunicação, Saúde, Educação 2000;4(7):71-80.

6. Soares IO. Educomunicação: quando pesquisa, extensão e ensino se embrigam!. Revista Comunicação \& Educação 
2013;18(1):7-14.

7. Barreto, RG. Tecnologias na formação de professores: o discurso do MEC. Educação e Pesquisa 2003;29(2):271-286.

8. Droescher, FD; Silva, EL. O pesquisador e a produção científica. Perspectivas em Ciência da Informação 2014;19(1):170-189.

9. Caldas G. Mídia e políticas públicas para a comunicação da ciência. In: Porto CM, Brotas AMP, Bortoliero ST, orgs. Diálogos entre ciência e divulgação científica: leituras contemporâneas [online]. Salvador: EDUFBA; 2011. 19-36p.

10. Rodrigues C; Blattmann U. Gestão da informação e a importância do uso de fontes de informação para geração de conhecimento. Perspectivas em Ciência da Informação 2014;19(3):4-29.

11. Xavier C. Mídia e saúde, saúde na mídia. In: Santos A.
Caderno mídia e saúde pública. Belo Horizonte: Escola de Saúde Pública/FUNED, 2006.

12. Taggart T, Grewe ME, Conserve DF, Gliwa C, Roman Isler M. Social Media and HIV: A systematic review of uses of social media in HIV communication. J Med Internet Res 2015;17(11) e248. DOI: http://dx.doi.org/10.2196/jmir.4387.

13. Castiel, LD. Insegurança, ética e comunicação em saúde pública. Rev Saude Publica 2003;37(2):161-167.

14. Figueira Júnior AJ. Potencial da mídia e tecnologias aplicadas no mecanismo de mudança de comportamento, através de programas de intervenção em atividade física. Rev. Bras. Ciên. e Mov, Brasília 2000;8(3):39-46.

15. Oliveira EBPM, Noronha DP. A comunicação científica e o meio digital. Inf. \& Soc.: Est. João Pessoa. 2005 jan./ jun;15(1):75-92 . 